\title{
Absence of leukocytosis in bacteraemic pneumococcal pneumonia
}

\author{
*Victoria Furera, David Raveh", Elie Picard', Shmuel Goldberg', Gabriel Izbickic \\ a Department of Internal Medicine, Shaare Zedek Medical Center, Jerusalem, Israel

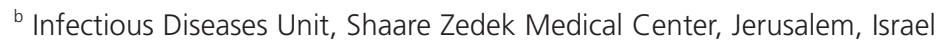 \\ ' Institute of Pulmonology, Shaare Zedek Medical Center, Jerusalem, Israel
}

Received 28th April 2010; revised 12th December 2010; accepted 31st December 2010; online 21st April 2011

\begin{abstract}
Background: Evaluation of patients with respiratory symptoms in primary care medicine is often based on peripheral WBC count that dictates the extent of diagnostic investigation. A normal WBC count may result in a limited investigation, often omitting chest radiography.

Aims: To determine the extent to which patients hospitalised with bacteraemic pneumococcal pneumonia have no leukocytosis at presentation.

Methods: A retrospective analysis was performed of patients with bacteraemic community-acquired pneumococcal pneumonia from 2000 to 2007 in a community care academic medical centre. Records were reviewed for symptoms, signs, and laboratory data including pneumococcal serotypes, chest radiographs on admission, and outcome.

Results: $21 \%$ of the patients presented with a normal WBC count (16.7\% of the children and $25.6 \%$ of the adults). Among this population with a normal WBC count at presentation, $90 \%$ of the adults and $70 \%$ of the children developed leukocytosis within a few days after admission.

Conclusions: In this study, in as many as one-fifth of all the patients with bacteraemic pneumococcal pneumonia, there was no leukocytosis at presentation. We therefore suggest that every patient with clinically suspected pneumonia should undergo chest radiography even if the WBC count is normal.

(c) 2011 Primary Care Respiratory Society UK. All rights reserved.

V Furer et al. Prim Care Respir J 2011; 20(3): 276-281

http://dx.doi.org/10.4104/pcrj.2011.00023
\end{abstract}

Keywords pneumonia, infection, diagnosis, management, WBC count, leukocytosis

\section{Introduction}

Community-acquired pneumonia (CAP) is a common and serious illness with considerable morbidity and mortality. ${ }^{1}$ An estimated 5.6 million cases of CAP occur annually in the United States, ${ }^{2}$ with 1.2 million patients hospitalised and an inpatient mortality rate of $5.8 \% .{ }^{3}$ Streptococcus pneumoniae accounts for about $50 \%$ of all cases of CAP requiring admission to hospital. ${ }^{4}$ Diagnostic evaluation of patients with symptoms suggestive of pneumonia is important and quite common, especially in the primary care setting. Since the presenting symptoms are often non-specific, the accurate assessment leading to a diagnosis of CAP should be pursued and followed by the appropriate assessment of illness severity. The diagnosis of CAP is suggested by the presence of non-specific clinical features (e.g. cough, fever, purulent sputum, and sometimes pleuritic chest pain) along with auscultatory findings, and is confirmed by imaging of the lungs, usually by chest radiography. ${ }^{5,6}$ Current clinical practice is still frequently based on the peripheral white blood cell (WBC) count in evaluating patients presenting with respiratory symptoms. While a finding of leukocytosis prompts a physician to obtain a chest radiograph, a normal WBC count supports a less extensive investigation, often omitting chest radiography, sending serology for atypical pneumonia, and even starting inappropriate treatment.

\footnotetext{
* Corresponding author: Dr Gabriel Izbicki, Institute of Pulmonology, Shaare Zedek Medical Center, Bayit Street, POB 3235, Jerusalem, 91031 Israel. Tel: 00-972-2-6666278 Fax: 00-972-2-6666772 E-mail: izbicki@szmc.org.il
} 
In patients with pneumococcal pneumonia the peripheral WBC count generally exceeds $11,000 / \mathrm{mm}^{3}, 7,8$ although it can be lower than $6,000 / \mathrm{mm}^{3}$ in $5-10 \%$ of patients. Such a low count has been found to be strongly associated with a poor prognosis. $^{8-10}$

It is not known what proportion of patients with bacteraemic pneumococcal pneumonia present with a normal WBC count and thus might have a deferred or incorrect diagnosis and treatment.

The aim of this study was to determine the percentage of inpatients with bacteraemic pneumococcal CAP presenting without leukocytosis to an emergency department and to characterise this group of patients.

\section{Methods}

\section{Study subjects and data collection}

The study was retrospectively conducted in a 550-bed community care university hospital. The charts of all patients discharged with diagnoses of 'pneumococcal pneumonia' (ICD code 481) or 'pneumonia and pneumococcal bacteremia' (ICD codes 486 and 041.2) from 1 January 2000 to 31 December 2007 were reviewed.

Community-acquired pneumococcal pneumonia was defined as symptoms of the lower respiratory tract along with new infiltrates seen on a chest radiograph and the presence of blood culture-proven pneumococcal infection. Exclusion criteria were immunocompromised patients, haematological or oncological disease and systemic steroid treatment.

The following data were collected: demographic characteristics, pre-existing co-morbid medical conditions, smoking status, initial vital signs, routine laboratory test results, pneumococcal serotypes isolated from blood cultures, and outcome of the hospitalisation with special mention of ventilation. The pneumonia severity index was calculated according to Patient Outcome Research Team (PORT) criteria for all adult patients. ${ }^{11}$

According to our laboratory reference values, leukocytosis was defined as $\geq 10,000$ cells $/ \mathrm{mm}^{3}$. For the purpose of this study, mild leukocytosis was defined as 10,000-15,000 cells $/ \mathrm{mm}^{3}$, significant leukocytosis as 15,000-25,000 cells $/ \mathrm{mm}^{3}$, and extreme leukocytosis (leukaemoid reaction) as $\geq 25,000$ cells $/ \mathrm{mm}^{3}$.

Approval from the Institutional Review Board was waived as this was a retrospective study with chart analysis.

\section{Statistical analysis}

Data were typed into an Excel spreadsheet (Microsoft, USA) and analysed by Epilnfo 3.5.1 (CDC, Atlanta, USA); Stata 9.0 (StataCorp, Texas, USA) was used for logistic regression analysis. We applied the $\chi^{2}$ test (Fisher exact test where applicable) for categorical variables and the two-tailed non-paired $t$ test for continuous variables.

For the purpose of our analysis, children were defined as subjects $<18$ years of age and adults were defined as subjects $\geq 18$ years of age.

\section{Results}

During an 8-year period, 120 patients with encoded diagnoses of 'pneumococcal bacteremia' (ICD code 041.2) and 'pneumonia' (ICD code 486) ( $n=17)$ or 'pneumococcal pneumonia' (ICD code 481) $(n=103)$ were identified in our computerised hospital's diagnosis data. After reviewing the charts, 39 cases were excluded: 35 failed to meet the established diagnostic inclusion criteria due to either erroneous coding or an absence of blood culture-proven pneumococcal bacteraemia and four had an underlying malignancy. No patients were immunosuppressed or on steroid treatment. Thus, 81 patients with bacteraemic pneumococcal pneumonia were included in the study. There were no nosocomial cases.

The baseline characteristics of the patient population are presented in Table 1.

All children and the majority (80\%) of the adults were non-smokers. In the adult group, $20 \%$ of patients were current smokers, $10 \%$ reported smoking in the past, and in $10 \%$ the smoking status was not reported. Almost $30 \%$ of the children were treated with antibiotics prior to hospitalisation compared with $2.5 \%$ of the adults.

The mean PORT score at admission was calculated in the entire adult group except for one case in which some data were unavailable. No cases were in PORT class I as these patients are usually treated on an outpatient basis; 33\% of patients were in the lower PORT scale (II and III) while the majority (70\%) were in classes IV and V. Seven patients (8.6\%) died during hospitalisation, all of whom were adults. In these cases the PORT score on admission ranged from 138 to 219 (class V). The mean \pm SD hospital stay was $6.0 \pm 4.6$ days for children and 10.6 \pm 12.6 for adults.

As anticipated, higher rates of co-morbidities were found in adults than in children. Three children had heart failure: two cases were associated with atrioventricular canal defects (in patients with Down syndrome) and one case with atrial septal defect (Holt Oram syndrome). Lung disease was detected in four subjects, two of whom were the aforementioned patients (one with Down syndrome and the other with Holt Oram syndrome), while the two others had asthma. The hospital course was complicated with empyema and drainage in eight children and four adults. Three of the eight children and one of the four adults with empyema had a normal WBC count at presentation. They all developed a high WBC count during their hospitalisation course. The maximal WBC count developed in children with empyema without leukocytosis at presentation at $4.7 \pm 0.6$ days and in adults with empyema at $7 \pm 0$ days after admission. The average hospital stay in patients with empyema was 
Table 1. Baseline characteristics of the patient population*

\begin{tabular}{|c|c|c|}
\hline & $\begin{array}{l}\text { Children } \\
\text { (<18 yrs) }\end{array}$ & $\begin{array}{l}\text { Adults } \\
(\geq 18 \mathrm{yrs})\end{array}$ \\
\hline Number & 42 & 39 \\
\hline Mean age (years) & $2.4 \pm 2.2$ & $55.8 \pm 23.2$ \\
\hline Age range (years) & $0.4-9$ & $21-94$ \\
\hline Male sex (\%) & 54.8 & 53.9 \\
\hline Smoker (\%) & 0 & 20.1 \\
\hline Past smoker (\%) & 0 & 10.2 \\
\hline Previous antibiotic use (\%) & 28.6 & 2. 6 \\
\hline \multicolumn{3}{|l|}{ Coexisting conditions (\%) } \\
\hline Congestive heart failure & 7.1 & 10.3 \\
\hline Lung disease & 9.5 & 18.0 \\
\hline Cerebrovascular disease & 0 & 18.0 \\
\hline Diabetes mellitus & 0 & 5.1 \\
\hline Renal disease & 2.4 & 12.9 \\
\hline Liver disease & 0 & 2.56 \\
\hline \multicolumn{3}{|l|}{ Physical examination } \\
\hline Temperature $\left({ }^{\circ} \mathrm{C}\right)$ & $38.5 \pm 1.1$ & $37.5 \pm 1.4$ \\
\hline Room air saturation (\%) & $92 \pm 4$ & $88 \pm 10$ \\
\hline Systolic blood pressure & $104 \pm 13$ & $119 \pm 26$ \\
\hline Diastolic blood pressure & $62 \pm 13$ & $66 \pm 19$ \\
\hline Pulse rate & $156 \pm 17$ & $107 \pm 22$ \\
\hline \multicolumn{3}{|l|}{ Laboratory results } \\
\hline Haematocrit (\%) & $32.9 \pm 4.6$ & $39.6 \pm 5.7$ \\
\hline Sodium (mEq/L) & $135.6 \pm 4$ & $136 \pm 6.8$ \\
\hline Creatinine $(\mathrm{mg} / \mathrm{dL})$ & $0.4 \pm 0.2$ & $1.9 \pm 1.5$ \\
\hline Glucose (mg/dL) & $109.7 \pm 18.6$ & $133.7 \pm 43.7$ \\
\hline $\mathrm{pH}$ & $7.39 \pm 0.1$ & $7.33 \pm 0.1$ \\
\hline \multicolumn{3}{|c|}{ PORT score ( $\%$ of the number of adult group)t } \\
\hline Class II & NA & 12.8 \\
\hline Class III & NA & 20.5 \\
\hline Class IV & NA & 28.2 \\
\hline Class V & NA & 35.9 \\
\hline Length of hospitalisation (days) & $6.0 \pm 4.6$ & 10. $6 \pm 12.6$ \\
\hline
\end{tabular}

prolonged $(11.25 \pm 2.25$ days in children and $15.5 \pm 5$ days in adults), as anticipated in complicated pneumonia cases.

In the adult group, three females were in the third trimester of pregnancy (ranging from 35 to 37 weeks). One of them had a normal WBC count on admission while the other two presented with mild and significant leukocytosis, respectively.

Physical examination findings and main blood test results are shown in Table 1. While more than half of the children
$(57.1 \%)$ presented with high fever $\left(>38.5^{\circ} \mathrm{C}\right)$, only $20.5 \%$ of the adults were febrile at admission. Moreover, five patients (three children and two adults) of the 81 (6.2\%) were afebrile and with normal WBC count at presentation. As we have no information on whether or not these patients received antipyretic drugs prior to hospitalisation, this observation is to be taken with circumspection. Room air oxygen saturation $<90 \%$ was noted in $26.2 \%$ of children and in $35.9 \%$ of the adult group. No significant statistical difference was found between the clinical and laboratory data of patients presenting with and without leukocytosis.

Table 2 shows the WBC cell count divided into three groups - normal WBC, mild and significant leukocytosis at presentation - in children and adults.

Leukocytosis was marked at presentation in the majority of patients. While 13\% (5\% children and $8 \%$ adults) developed only mild leukocytosis, 51\% (30\% children and $21 \%$ adults) developed significant leukocytosis. The average length of hospital stay in children with mild leukocytosis $(n=5)$ and in those with significant leukocytosis $(n=30)$ was 8.0 and 5.2 days, respectively. In the adult group the average length of hospital stay was longer than in children (9.3 days in the group presenting with mild leukocytosis $(n=8)$ and 10.6 days in patients with significant leukocytosis $(n=21))$.

Twenty-one percent of all patients (16.7\% of children and $25.6 \%$ of adults) had a normal WBC count on admission. Most of the adults (90\%) presenting with a normal WBC count developed leukocytosis later during their hospitalisation: 50\% developed mild leukocytosis and $40 \%$ developed significant leukocytosis. Half of the adult patients with mild leukocytosis at presentation developed significant leukocytosis later on during the hospitalisation. The maximal WBC count was observed $5.2 \pm 5.1$ days after hospitalisation in the adult group.

In the paediatric population, $71 \%$ of patients presenting with a normal WBC count developed leukocytosis during their hospitalisation. The highest WBC count appeared within $4.0 \pm 1.7$ days of hospitalisation.

By multivariate analysis, no variables were found to be associated with the absence of leukocytosis at presentation.

When analysing a subgroup of geriatric patients aged $>65$ years ( 15 cases), we found that $20 \%$ presented with a normal WBC count and another 20\% presented with extreme leukocytosis, compared with $29 \%$ and $37.5 \%$, respectively, in the non-geriatric group.

Seven of the 81 patients $(8.6 \%)$ died during hospitalisation. All the other patients were discharged from hospital in a stable condition.

\section{Serogroup distribution}

Eighteen different serotypes were found among 67 patients (36 children and 31 adults), but only five of them accounted for $73.1 \%$ of the strains. Serotypes were unavailable for 14 
Table 2. White blood cell count at presentation according to age group, length of hospital stay, and outcome

\begin{tabular}{|c|c|c|c|c|}
\hline & \multicolumn{3}{|c|}{ White blood cell count $/ \mathrm{mm}^{3}$} & \multirow[t]{2}{*}{$p$ value } \\
\hline & $<10,000$ & $10,000-15,000$ & $>15,000$ & \\
\hline No $(\%)$ children $(n=42)$ & $7(16.7)$ & $5(11.9)$ & $30(71.4)$ & NS (0.26) \\
\hline Hospital stay, children (days) & $8.0 \pm 4.6$ & $8.0 \pm 5.1$ & $5.2 \pm 4.6$ & NS $(0.21)$ \\
\hline Hospital stay, adults (days) & $11.6 \pm 13.9$ & $9.3 \pm 12.7$ & $10.6 \pm 12.7$ & NS (0.46) \\
\hline Dead & $1(10 \%)$ & $2(25 \%)$ & $4(19 \%)$ & \\
\hline
\end{tabular}

patients. The five serotypes found most frequently were 1 $(23.9 \%), 5(20.9 \%), 14(16.4 \%), 6 \mathrm{~B}(7.4 \%)$, and $7 \mathrm{~F}(4.5 \%)$. The serotype distribution was similar in both genders. Serotype 14 was ranked first in frequency in children (30.5\%) and was exclusive for this population. In the elderly population, serotype $7 \mathrm{~F}$ was associated with more severe disease at presentation, supported by higher PORT scores. There was no correlation between pneumococcal serotype and WBC count at presentation and/or outcome.

\section{Discussion}

CAP is a common disorder which is potentially life-threatening, especially in older adults and patients with co-morbid diseases. Despite all rigorous definitions of pneumonia requiring the finding of a pulmonary infiltrate on a chest radiograph, ${ }^{5,6,12-14}$ in daily practice in patients with mild respiratory symptoms and a normal WBC count a chest radiograph will often not be ordered.

In this study we found that $21 \%$ of patients with bacteraemic pneumococcal pneumonia presented with a normal WBC count ( $16.7 \%$ of children and $25.6 \%$ of adults). These findings are consistent with one previously published study. ${ }^{8}$ This detailed analysis of adult bacteraemic pneumococcal pneumonia in a community teaching hospital during the years 1992-1996 found that, among 108 patients, only 73.2\% presented with a leukocyte count $>11,000$ cells $/ \mathrm{mm}^{3}$. The authors noted that leukocytosis was associated with a better prognosis than a normal leukocyte count and leukopenia, ${ }^{8,15}$ findings that were not confirmed in our study.

\section{Paediatric subgroup}

Most children with bacteraemic pneumococcal pneumonia have a typical illness with high temperature, leukocytosis and lobar or segmental consolidation on the chest radiograph. 8,13 However, as many as 30\% of patients might have an atypical illness, demonstrating the clinical variability of bacteraemic pneumonia. ${ }^{7}$ As shown previously by Toikka et al. ${ }^{7}$ and confirmed in our study, $17 \%$ of children presented with normal WBC on admission. Since our study combined both paediatric and adult populations, we used the definition of leukocytosis $\left(>10,000\right.$ cells $/ \mathrm{mm}^{3}$ ) as applicable in the adult population. Although in the paediatric population (mainly aged $<6$ years) leukocytosis is usually defined as a WBC count $\geq 15,000$ cells $/ \mathrm{mm}^{3}, 7,13$ we decided to define leukocytosis according to the adult values so it is possible that even more children would have presented with normal leukocytosis if the adjusted paediatric values had been used.

\section{Geriatric subgroup}

In the geriatric population a diagnosis of pneumonia might be challenging since the symptoms and signs of the infection are often incompletely expressed. ${ }^{16}$ In our study, analysis of the geriatric subpopulation showed that normal and extreme WBC counts at presentation occurred equally ( $20 \%$ in each group).

An observational study of pneumococcal pneumonia in 70 patients aged $\geq 80$ years showed that pleuritic pain was less frequent in this age group. ${ }^{17}$ Nevertheless, in our study $60 \%$ of this group presented as classical bacteraemic pneumonia syndrome, defined as the presence of at least three of the following symptoms: acute chest pain, chills, pleuritic chest pain, and purulent sputum. ${ }^{17}$ Advanced age is known to be associated with reduced symptoms. ${ }^{18}$ Febrile responses to infectious diseases in geriatric patients are often blunted or absent. ${ }^{19}$ Leukocytosis and an excess of immature WBC (bandemia) are generally reported less frequently in older patients, ${ }^{16}$ although some observational studies point out that the majority of elderly patients with $\mathrm{CAP}^{20}$ or community-acquired bacteraemic infections tend to develop leukocytosis on presentation. ${ }^{21}$ Given the higher frequency of a presentation with non-specific pneumonia in elderly patients, underdiagnosis of this illness can occur, leading potentially to a high rate of complications and death. The mortality rate of CAP doubles as age increases, from $7.8 \%$ in those aged $65-69$ years to $15.4 \%$ in those aged $\geq 90$ years. ${ }^{22}$ Our study supports the practice of not focussing on the WBC count in the context of a possible pneumonia.

\section{Serotype analysis}

The pneumococcal strain survey showed that serotype 14 was the most common serotype in the paediatric group and exclusive to it. This finding is consistent with data summarised 
from 35 studies reported from 16 countries in Western Europe which found that serotype 14 was the most common in a patient population aged $<18$ years, ranging from $19 \%$ to $47 \% .{ }^{23}$ This strain is included in Prevnar, a heptavalent pneumococcal conjugate vaccine (PCV7) approved by the FDA in February 2003. None of the children in our cohort received the Prevnar vaccine, and this result therefore reinforces the importance of this vaccination in our population. ${ }^{24}$ Serotype $7 F$ was restricted to the adult group, being associated with more severe morbidity in the elderly. This serotype is included in the polyvalent pneumococcal vaccine (Pneumovax) which is widely available in most countries. No data were available regarding the Pneumovax immunisation status of the study subjects so we do not know whether the infected cases represented non-immunised patients or cases of vaccine failure.

\section{Guidelines}

The need for chest radiography in febrile children is not clear in the literature. One study showed that $25 \%$ of young children with a high temperature, WBC count $>20,000 / \mathrm{mm}^{3}$ and no clinical signs of respiratory distress had radiographic signs of pneumonia. ${ }^{25}$ In contrast, another study showed that very few infants with fever but no respiratory signs had pneumonia. ${ }^{26}$ The British Thoracic Society (BTS ${ }^{27}$ recommends that chest radiography should be considered in young children with pyrexia of unknown origin without reference to the WBC unless features of bronchiolitis are present. The BTS guidelines for the management of CAP in adults (updated in 2009) ${ }^{28}$ state that it is not necessary to perform a chest $x$-ray in patients with suspected CAP unless the diagnosis is in doubt or the progress following treatment for CAP is not satisfactory. However, the guidelines state that all patients admitted to hospital with suspected CAP should have a chest $x$-ray performed as soon as possible to confirm or refute the diagnosis.

\section{Limitations of the study}

Our study closely examines-cases of proven pneumococcal pneumonia encompassing a whole range of ages, focusing on the laboratory findings on presentation and over the hospitalisation course. While a retrospective observation allows a precise isolation of the particular disorder, this study design also has a few limitations. First, the sample of the study was relatively small. Second, only hospitalised patients were included, suggesting a bias towards more severe cases. Finally, because we only included patients with positive culture bacteraemia, those with non-bacteraemic pneumococcal pneumonia or non-pneumococcal pneumonia were omitted.

\section{Conclusions}

In this study of 81 hospitalised patients with bacteraemic pneumococcal pneumonia, $16.7 \%$ of the children and $25.6 \%$ of the adults had no leukocytosis at presentation. There was no predictive factor for presenting without leukocytosis. We therefore suggest that every patient with a clinical suspicion of pneumonia should undergo a chest x-ray, even if the WBC count is in the normal range.

\section{Conflicts of interest}

None.

\section{Funding}

None.

\section{References}

1. Feikin DR, Schuchat A, Kolszak M, et al. Mortality from invasive pneumococcal pneumonia in the era of antibiotic resistance, 1995-1997. Am J Public Health 2000;90:223-9. http://dx.doi.org/10.2105/AJPH.90.2.223

2. Bartlett JG, Dowell SF, Mandell LA, et al. Practice guidelines for the management of community-acquired pneumonia in adults. Infectious Diseases Society of America. Clin Infect Dis 2000;31:347-82. http://dx.doi.org/10.1086/313954

3. Lindenauer PK, Behal R, Murray CK, et al. Volume, quality of care, and outcome in pneumonia. Ann Intern Med 2006;144:262-9.

4. Almirall J, Bolibar I, Vidal J, et al. Epidemiology of community-acquired pneumonia in adults: a population based study. Eur Respir J 2000;15:757-63. http://dx.doi.org/10.1034/j.1399-3003.2000.15d21.x

5. Mandell LA, Wunderink RG, Anzueto A, et al. Infectious diseases society of America/American thoracic society consensus guidelines on the management of community-acquired pneumonia in adults. Clin Infect Dis 2007;44:\$27-72. http://dx.doi.org/10.1086/511159

6. Mandell LA, Marrie TJ, Grossman RF, et al. Canadian guidelines for the initial management of community-acquired pneumonia: an evidence-based update by the Canadian Infectious Diseases Society and the Canadian Thoracic Society. Clin Infect Dis 2000;31:383-421. http://dx.doi.org/10.1086/313959

7. Toikka P, Virki R, Mertsola J, et al. Bacteremic pneumococcal pneumonia in children. Clin Infect Dis 1999;29:568-72. http://dx.doi.org/10.1086/598635

8. Watanakunakorn C, Bailey TA. Adult bacteremic pneumococcal pneumonia in a community teaching hospital, 1992-1996. Arch Intern Med 1997;157:196571.

9. Musher DM, Alexandraki I, Graviss EA, et al. Bacteremic and nonbacteremic pneumococcal pneumonia: a prospective study. Medicine 2000;79:210-21. http://dx.doi.org/10.1097/00005792-200007000-00002

10. Martens P, Worm SW, Lundgren B, et al. Serotype-specific mortality from invasive Streptococcus pneumoniae disease revisited. BMC Infect Dis 2004;4:21. http://dx.doi.org/10.1186/1471-2334-4-21

11. Fine MJ, Auble TE, Yealy DM, et al. A prediction rule to identify low-risk patients with community acquired pneumonia. N Engl J Med 1997;336:243-50. http://dx.doi.org/10.1056/NEJM199701233360402

12. Metlay JP, Kapoor WN, Fine MJ. Does the patient have community-acquired pneumonia? Diagnosing pneumonia by history and physical examination. JAMA 1997;278:1440-5.

13. Rahav G, Toledano $Y$, Engelhard G, et al. Invasive pneumococcal infections. A comparison between adults and children. Medicine 1997;76:295-303. http://dx.doi.org/10.1097/00005792-199707000-00007

14. Franquet T. Imaging of pneumonia: trends and algorithms. Eur Respir J 2001;18:196-208.

15. Watanakunakorn C, Greifenstein A, Stroh K, et al. Pneumococcal bacteremia in three community teaching hospitals from 1980-1989. Chest 1993;103:710-16. http://dx.doi.org/10.1378/chest.103.4.1152

16. Fein AM. Pneumonia in the elderly. Special diagnostic and therapeutic considerations. Med Clin North Am 1994;78:1015-32.

17. Fernández-Sabé N, Carratalà J, Rosón B, et al. Community-acquired pneumonia in very elderly patients: causative organisms, clinical characteristics, and 
outcomes. Medicine 2003;82:159-69. http://dx.doi.org/10.1097/00005792200305000-00002

18. Harper C, Newton P. Clinical aspects of pneumonia in the elderly veteran. J Am Geriatr Soc 1989:37:867-72.

19. Gleckman R, Hibert O. Afebrile bacteremia. A phenomenon in geriatic patients. JAMA 1982;248:1478-82.

20. Metlay JP, Schulz R, Li YH, et al. Influence of age on symptoms at presentation in patients with community-acquired pneumonia. Arch Intern Med 1997;153:1453-9.

21. Lee CC, Chen SY, Chang IJ, et al. Comparison of clinical manifestations and outcome of community-acquired bloodstream infections. Medicine 2007;86:138-44. http://dx.doi.org/10.1097/SHK.0b013e318067da56

22. Kaplan V, Angus DC, Griffin MF, et al. Hospitalized community-acquired pneumonia in the elderly: age- and sex-related patterns of care and outcome in the United States. Am J Respir Crit Care Med 2002;165:766-72.

23. Jefferson T, Ferroni $E$, Curtale $F$, et al. Streptococcus pmeumoniae in Western
Europe: serotype distribution and incidence in children less than 2 years old. Lancet Infect Dis 2006;6:405-10. http://dx.doi.org/10.1016/S14733099(06)70520-5

24. Preventing Pneumococcal Disease among Infants and Young Children. Recommendations of the Advisory Committee on Immunization Practices, CDC, MMWR 2000;49(RR09):1-38. Available at http://www.cdc.gov/mmwr/preview/mmwrhtml/rr4909a1.htm

25. Bachur R, Perry H, Harper MB. Occult pneumonia: empiric chest radiographs in febrile children with leukocytosis. Ann Emerg Med 1999;33:166-73. http://dx.doi.org/10.1016/S0196-0644(99)70390-2

26. Heulitt MJ, Ablow RC, Santos CC, et al. Febrile infants less than 3 months old: value of chest radiography. Radiology 1988;167:135-7.

27. British Thoracic Society guidelines for the management of community acquired pneumonia in childhood. Thorax 2002;57:11-i24.

28. British Thoracic Society guidelines for the management of community acquired pneumonia in adults: update 2009. Thorax 2009:64:11-i55.

Available online at http://www.thepcrj.org

\section{For more information and to register visit www.pcrs-uk.org/conferences/index.php}

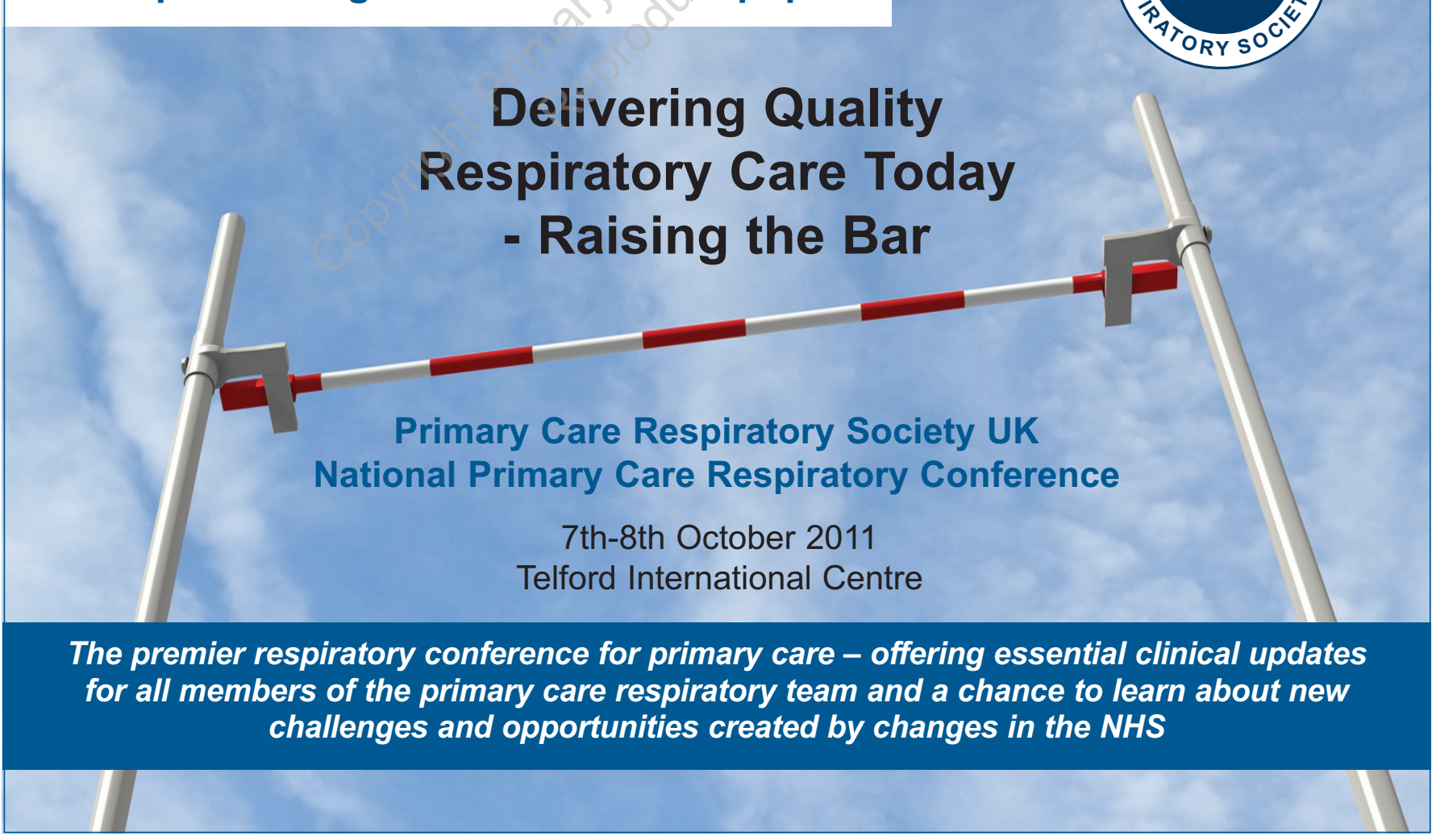

